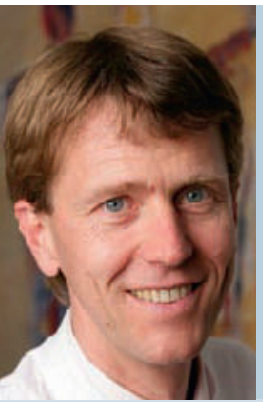

Prof. Dr. med. Heribert Schunkert Lübeck, Kardiologie

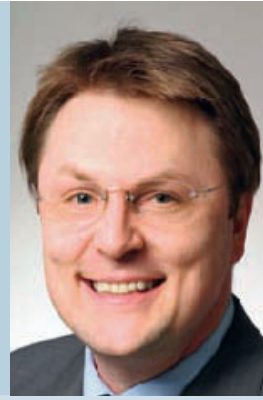

Prof. Dr. med. Jochen Seißler München, Diabetologie



Prof. Dr. med. Alfred Wirth Bad Rothenfelde, Adipositas

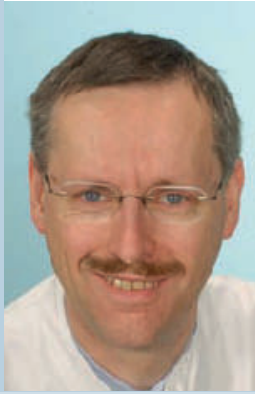

Prof. Dr. med. Walter Zidek

Berlin, Hypertensiologie

\title{
Mitralklappeninsuffizienz
}

\section{Perkutane Intervention oder Operation?}

Mit dem MitraClip steht ein perkutaninterventionelles Verfahren zur Therapie der Mitralklappeninsuffizienz zur Verfügung. In der EVEREST-II-Studie wurde der MitraClip mit der konventionellen Klappenoperation verglichen.

In der EVEREST-II-Studie wurden 279 Patienten mit moderater oder schwerer Mitralklappeninsuffizienz auf ein operatives oder perkutan-interventionelles Vorgehen (MitraClip) randomisiert. Der kombinierte primäre Endpunkt bestand aus dem ereignisfreien Überleben (ohne Tod, Mitralklappenoperation, Mitralklappeninsuffizienz $3+/ 4+)$ innerhalb von zwölf Monaten nach der Prozedur. 55\% der Patienten in der perkutan-interventionellen und $73 \%$ in der operativen Gruppe erreichten den primären Endpunkt $(p=0,007)$. Bei gleicher Letalität in beiden Gruppen (je 6\%) war dieser Unterschied vor allem durch die Notwendigkeit zum operativen Vorgehen aufgrund einer weiter oder erneut bestehenden Mitralklappeninsuffizienz in der MitraClip-Gruppe bedingt. Interessant war vor allem die Beobachtung, dass einige Subgruppen (Alter $\geq 70$ Jahre, funktionelle Mitralklappeninsuffizienz, linksventrikuläre Pumpfunktion $<60 \%$ ) hinsichtlich des primären Endpunkts keinen Vorteil bei einem primär chirurgischen Vorgehen hatten (Abb. 1).

Die Autoren folgerten, dass die perkutane Intervention unter Verwendung des MitraClip bei schwerer Mitralklappeninsuffizienz weniger effektiv in der Reduktion der
Mitralklappenregurgitation ist als die konventionelle chirurgische Vorgehensweise. Andererseits zeigten sich für die mit dem MitraClip behandelten Patienten weniger perioperative Komplikationen (Beatmungsdauer, Bluttranfusionen) sowie ein vergleichbares Belastungsniveau nach zwölf Monaten.

II Kommentar: Die Einführung und Weiterentwicklung perkutan-interventioneller Verfahren zur Therapie erworbener Herzklappenfehler ist ein bedeutender und unumkehrbarer Fortschritt in der kardiovaskulären Medizin. Schon heute profitieren viele Patienten mit schwerer Aortenklappenstenose und Komorbiditäten vom perkutanen Klappenersatz (TAVI). Für den MitraClip wurde nun ebenso gezeigt, dass er für ausgesuchte Patienten ein alternatives Verfahren zur chirurgischen Therapie darstellt.
Eine fortlaufende technische Verbesserung der Methoden ist zu erwarten. Die Ausweitung des Indikationsspektrums bei Mitralklappeninsuffizienz gegenüber einem medikamentös-konservativen oder einem operativen Vorgehen wird Gegenstand zukünftiger Studien sein. Auch aufgrund der zunehmenden Anzahl und Komplexität zur Verfügung stehender Therapiemaßnahmen bei strukturellen Herzerkrankungen erscheint jedoch eine noch engere interdisziplinäre Kooperation vor allem zwischen der Kardiologie und der Kardiochirurgie notwendig.

Prof. Dr. med. Peter W. Radke,

Universitätsklinikum Schleswig-Holstein, Campus Lübeck II

II Feldman T et al. Percutaneous Repair or Surgery for Mitral Regurgitation. N Engl J Med 2011;364:1395-1406

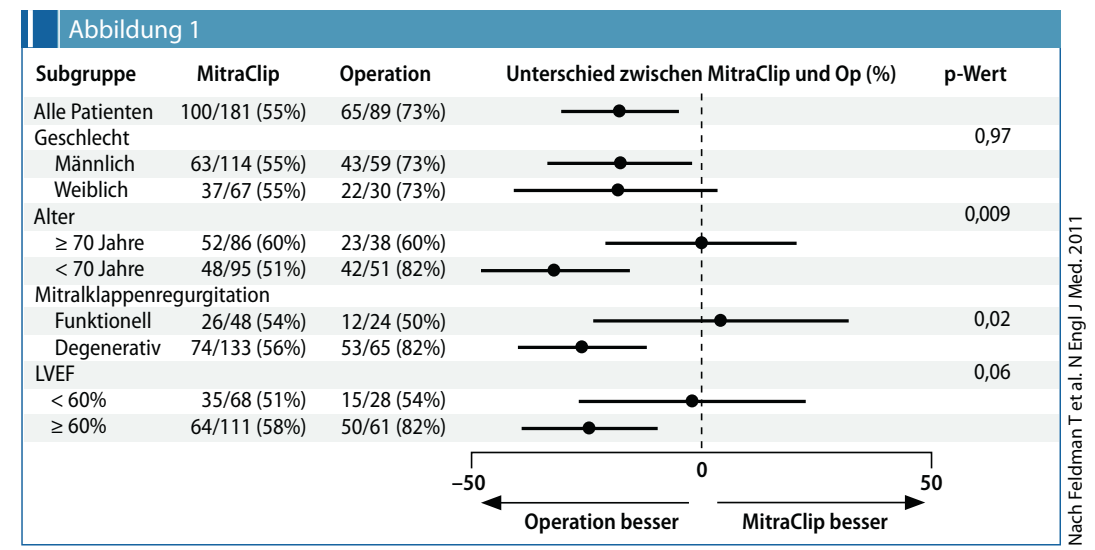

